\title{
Research organizations under scrutiny new indicators and analytical results
}

Selected papers presented at the "20th International Conference on Science and Technology Indicators" held in Lugano (Switzerland) on 2-4 September 2015

Guest Editors:

Benedetto Lepori, Sybille Hinze, Wolfgang Glänzel 